\title{
Single-photon entanglement at acousto-optic (acousto-gyration) diffraction
}

\author{
Skab I., Kostyrko M. and Vlokh R. \\ O. G. Vlokh Institute of Physical Optics, 23 Dragomanov Street, 79005 Lviv, \\ Ukraine
}

Received: 16.07 .2020

\begin{abstract}
We show that a single-photon entanglement can be realized at the collinear acousto-gyration diffraction in optical active and optically uniaxial (or isotropic) media. The entanglement between two degrees of freedom, spin angular momentum and orbital angular momentum, can occur when a standing longitudinal acoustic wave is excited in the above media. This makes it possible to realize four maximum entangled Bell states.
\end{abstract}

Keywords: quantum entanglement, photons, optical activity, acousto-optics, acousto-gyration

UDC: $535.56+535.14+534.16+535.42$

\section{Introduction}

Quantum entanglement is one of the pronounced effects that make quantum mechanics different from it classical analogue [1]. This effect, which consists in non-classical correlations between distant quantum systems, has at first been criticized as an Einstein-Podolsky-Rosen paradox in the work [2]. A non-local character of quantum-mechanical effects becomes commonly perceived only after a well-known theorem by J. S. Bell has been formulated [3] and a series of appropriate experiments have been reported $[4,5]$.

The quantum entanglement has been observed using different particles such as ions [6], nuclei and electronic spins [7] and photons [8]. Preparation of entangled photons is probably the most simple from the viewpoint of experimental procedures. Moreover, the entangled photons have been used to implement quantum teleportation, quantum logic and quantum computation operations [9-13]. Classical experiments with correlated photons are based on such nonlinear optical phenomena as spontaneous parametric down-conversion [14], four-wave mixing [15] or cascade radiation in calcium excited due to a two-photon absorption [5, 16, 17]. During the nonlinear processes mentioned above, decay of one (or more) photon(s) into two (or more) photons with entangled quantum states can be realized. The outgoing photons (e.g., at the downconversion) can be described by the wave functions

$$
|\psi\rangle=\frac{1}{\sqrt{2}}\left(\left|\psi_{1}^{m}\right\rangle \otimes\left|\psi_{2}^{n}\right\rangle \pm\left|\psi_{1}^{n}\right\rangle \otimes\left|\psi_{2}^{m}\right\rangle\right)
$$

where $\left\{\left|\psi_{1}^{m}\right\rangle,\left|\psi_{2}^{m}\right\rangle\right\}$ and $\left\{\left|\psi_{1}^{n}\right\rangle,\left|\psi_{2}^{n}\right\rangle\right\}$ are the basis vectors in some Hilbert spaces $H_{m}$ and $H_{n}$. States of these photons are non-separable [18], i.e. the state vector of whole system

$$
|\psi\rangle=\left|\psi_{i}^{m}\right\rangle \otimes\left|\psi_{j}^{n}\right\rangle
$$

cannot be decomposed in the tensor product. Here the pure state is given by $|\psi\rangle \in H_{m} \otimes H_{n}$, while 
$\left|\psi_{i}^{m}\right\rangle$ and $\left|\psi_{j}^{n}\right\rangle$ are the pure states of $i$ and $j$ subsystems. In the case of two-particle entanglement, one can remind a simple criterion [18], which states that the particles are entangled when the determinant of tensor product of their states is not equal to zero $(\operatorname{det}|\psi\rangle \neq 0)$. This criterion represents an easy tool in analyzing the possibilities for realization of quantum entanglement.

So-called intrasystem [19] (or single-photon [20]) entanglement effects can be mentioned among the possibilities for realizing the quantum entanglement. Then a single photon reveals entanglement between its different degrees of freedom, e.g. spin angular momentum (SAM) and orbital angular momentum (OAM). Note that a non-locality property of a quantum system does not manifest itself in this classical type of entanglement.

The nonlinear optical processes that can lead to an alternative intersystem quantum entanglement are described by the third- or fourth-order nonlinear susceptibilities, $\chi_{i j k}^{(3)}$ or $\chi_{i j k l}^{(4)}$. It would be proper to remind in this respect that the effect of acousto-optic diffraction is a bilinear process which is just described by a fourth-order susceptibility:

$$
P_{i}^{\omega \pm \Omega}=\chi_{i j k l}^{(4)} e_{j k}^{\Omega} E_{l}^{\omega} .
$$

In Eq. (3), $E_{l}^{\omega}$ is the electric field of the incident optical wave at the frequency $\omega, e_{j k}^{\Omega}$ the mechanical strain caused by the acoustic wave at the frequency $\Omega$, and $P_{i}^{\omega \pm \Omega}$ the polarization of diffracted wave at the frequency $\omega \pm \Omega$. Notice that the incident photon does not decay into zeroand first-order diffracted photons at the acousto-optic diffraction (e.g., at the Bragg diffraction). Instead, it can exist in this or that quantum state with certain probability. Therefore, one could assume that the acousto-optic diffraction cannot be accompanied with the intersystem entanglement.

The present work is aimed at ascertaining the possibilities for realization of the intrasystem quantum entanglement under the condition of acousto-optic diffraction.

\section{Analysis}

We will start our consideration from the analysis of appearance of non-separable states at the down-conversion, as an example. Let $C$ represent the incident photon which is nonlinearly converted into photons with lower energies due to the processes $C=V_{1}+H_{2}$ or $C=H_{1}+V_{2}$ (see Fig. 1). The state vectors for the vertical and horizontal polarizations read as

$$
V=\left(\begin{array}{l}
0 \\
1
\end{array}\right), H=\left(\begin{array}{l}
1 \\
0
\end{array}\right) \text {. }
$$

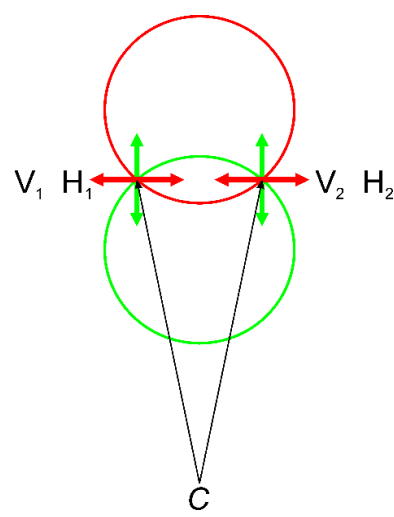

Fig. 1. Schematic view of photon states appearing at the down-conversion. Double-sided arrows indicate vertical $(\mathrm{V})$ and horizontal $(\mathrm{H})$ photon polarizations. 
The four maximum entangled Bell states in this case are as follows:

$$
|\psi\rangle_{1,2}=\frac{1}{\sqrt{2}}\left(\left|V_{1}\right\rangle \otimes\left|H_{1}\right\rangle \pm\left|H_{2}\right\rangle \otimes\left|V_{2}\right\rangle\right)=\frac{1}{\sqrt{2}}\left(\begin{array}{cc}
0 & \pm 1 \\
1 & 0
\end{array}\right), \quad \text { since } \operatorname{det}|\psi\rangle_{1,2}=\operatorname{det}\left(\begin{array}{cc}
0 & \pm 1 \\
1 & 0
\end{array}\right)=\mp 1,
$$

and

$$
|\psi\rangle_{3,4}=\frac{1}{\sqrt{2}}\left(\left|V_{1}\right\rangle \otimes\left|V_{2}\right\rangle \pm\left|H_{1}\right\rangle \otimes\left|H_{2}\right\rangle\right)=\frac{1}{\sqrt{2}}\left(\begin{array}{cc} 
\pm 1 & 0 \\
0 & 1
\end{array}\right), \text { since } \operatorname{det}|\psi\rangle_{3,4}=\operatorname{det}\left(\begin{array}{cc} 
\pm 1 & 0 \\
0 & 1
\end{array}\right)= \pm 1
$$

Notice that the equalities $\operatorname{det}\left(V_{1} V_{2}+V_{1} H_{1}\right)=\operatorname{det}\left(H_{2} V_{2}+H_{1} H_{2}\right)=\operatorname{det}\left(V_{1} V_{2}+H_{2} V_{2}\right)=$ $=\operatorname{det}\left(H_{1} H_{2}+V_{1} H_{1}\right)=0$ hold true and, hence, these states are separable. Eqs. (5) and (6) correspond to the two qubits that appear due to the down-conversion process.

Contrary to the down-conversion, in the case of anisotropic acousto-optic Bragg diffraction a photon can exist, with certain probabilities, in the two quantum states. These are zero and first diffraction orders which correspond to orthogonal light polarizations. Thus, one can consider either a single-photon positional-polarization intrasystem entanglement [21] (when the zero and first orders of diffraction are spatially separated) or a frequency-polarization intrasystem entanglement (when the zero and first orders of diffraction are frequency separated due to Doppler shift). Although, according to Ref. [21], these types of classical entanglement (i.e., incoherent superposition of the beams with orthogonal polarizations) are somewhat different from the quantum entanglement, still a closer case is the spatial-polarization type of entanglement which deals with the vector beams and the beams bearing OAM. Then a natural question arises: can the acousto-optic diffraction be accompanied by the appearance of OAM?

Let us now consider the effect of acousto-gyration diffraction, which is a particular kind of acousto-optic interactions in gyrotropic elasto-gyration media [22, 23]. As we have shown earlier $[22,23]$, propagation of longitudinal acoustic waves along an optic-axis direction in some optically uniaxial crystals, which exhibit elasto-gyration effect, can induce a diffraction grating based on the modulation of antisymmetric part of dielectric permittivity (or gyration tensor). This induces an acousto-gyration diffraction of light. The effect arises due to perturbations of dielectric permittivity via elasto-gyration coupling. Unlike the commonly known acousto-optic diffraction, the acousto-gyration can be only anisotropic, i.e. the incident and diffracted beams must have mutually orthogonal polarizations.

Let us examine a collinear case of interaction, when three interacting waves propagate along the optic axis in a uniaxial and optically active crystal (see Fig. 2). These are a longitudinal acoustic wave, an incident optical wave (e.g., a left-handed circularly polarized one), and a diffracted (right-handed circularly polarized) optical wave. Then the relation for the acousto-gyration figure of merit is defined as $M_{a g}=\frac{\beta_{33}^{2}}{\rho v_{33}^{3} n_{o}^{2}}$, with $\beta_{33}$ being the elasto-gyration coefficient, $v_{33}$ the longitudinal acoustic wave velocity (with its first index corresponding to the propagation direction and the second one to the polarization one), $n_{o}$ the ordinary refractive index, and $\rho$ the crystal density. We have found that $M_{a g}$ (e.g., for quartz crystals) is equal to $10^{-19}$ in its order of magnitude. In other words, the $M_{a g}$ parameter is four orders smaller than the typical figures of merit for the ordinary acousto-optic diffraction. It is obvious that the effect given by so a small size can hardly be detectable in practice. 

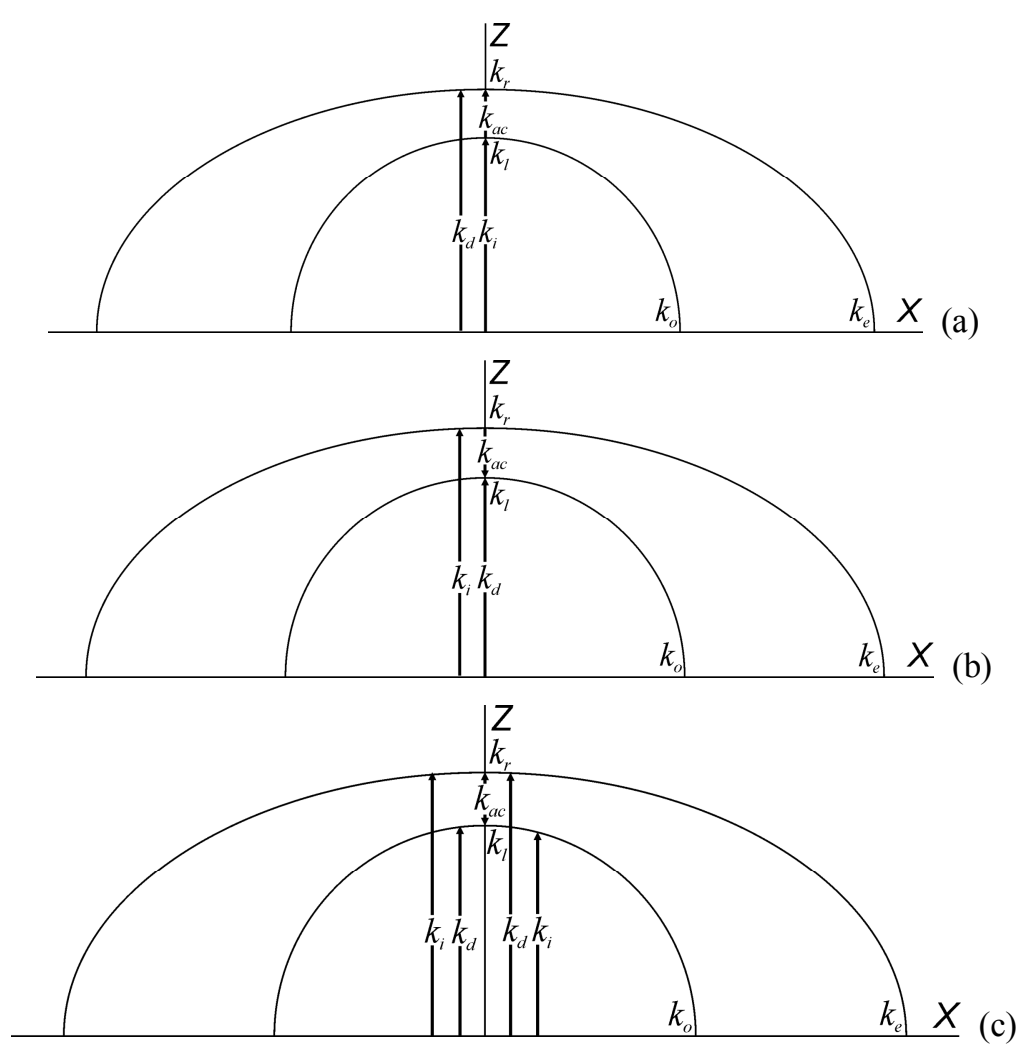

Fig. 2. Phase matching conditions for the acousto-gyration interactions that occur among a travelling (panels a and b) or standing (panel c) longitudinal acoustic wave and circularly (a, b) or linearly (c) polarized incident photons.

Nevertheless, the acousto-gyration diffraction can reveal very interesting peculiarities [24]. In fact, this effect manifests itself as a diffraction of, e.g., a right-handed optical photon (i.e., a photon with the SAM equal to $\sigma=\hbar$ ) at a longitudinal acoustic phonon with the zero total angular momentum. Then a left-handed diffracted photon (i.e., a photon with the SAM $\sigma=-\hbar$ ) appears. As a consequence, the SAM changes according to the scheme $\hbar \rightarrow-\hbar$. In such a case, the total angular momentum changes its value by $2 \hbar$. Since the principle of conservation of the total angular momentum requires equality of the momentums before the acousto-gyration interaction begins and after the process is completed, the diffracted photon must have an additional optical angular momentum equal to $2 \hbar$. The latter should obviously correspond to the OAM $l$, because the angular momentum cannot be transferred into mechanical one owing to the axial symmetry of interaction. This means that the circularly polarized diffracted photon must bear a doubly charged vortex and its quantum state has to be written as $|\sigma, l\rangle=|-\hbar, 2 \hbar\rangle$.

In the situation described above, one can distinguish the two Hilbert spaces corresponding to the spin and orbital degrees of freedom. However, only a collinearly diffracted wave has a nonzero OAM when being diffracted at a travelling acoustic wave (cf. Fig. 2a and Fig. 2b). Let us now imagine that some linearly polarized incident optical wave interacts with a standing longitudinal acoustic wave (see Fig. 2c). Then the incident wave will be decomposed into two circularly polarized waves with different polarization signs, which appear due to a circular optical birefringence. In other terms, the incident photon with a zero spin can exist, with the same 
probabilities, in two quantum states that differ by the signs of their SAM $(|\sigma\rangle=|R\rangle$ and $|-\sigma\rangle=|L\rangle)$. This state can be written as

$$
|\Psi\rangle=\frac{1}{\sqrt{2}}(|R\rangle+|L\rangle) .
$$

After the acoustic signal is switched on, the both quantum states of the photon will give rise to the diffracted photons in the quantum states $|\sigma, l\rangle=|-\hbar, 2 \hbar\rangle$ and $|\sigma, l\rangle=|\hbar,-2 \hbar\rangle$. Then the outgoing entangled state is as follows:

$$
|\Psi\rangle_{1}=\frac{1}{\sqrt{2}}(|L, 2 \hbar\rangle+|R,-2 \hbar\rangle) .
$$

Eq. (8) is written under the condition that the phase difference between the right-handed and lefthanded waves is compensated. Let the SAM belong to the Hilbert space with the basis vectors $n_{1}$ and $n_{2}$, while the OAM to the space with the basis vectors $m_{1}$ and $m_{2}$. Then the state given by Eq. (8) can be represented as

$$
|\Psi\rangle_{1}=\frac{1}{\sqrt{2}}\left|n_{1}, m_{2}\right\rangle+\frac{1}{\sqrt{2}}\left|n_{2}, m_{1}\right\rangle,
$$

which is encoded by the matrix

$$
B=\left|\begin{array}{cc}
0 & \frac{1}{\sqrt{2}} \\
\frac{1}{\sqrt{2}} & 0
\end{array}\right| .
$$

Notice that $\operatorname{det} B \neq 0$ and, according to the criterion mentioned above, this state is entangled. Let the phase difference between the right-handed and left-handed waves be equal to $\pi$. In this case, Eq. (8) can be rewritten to

$$
|\Psi\rangle_{2}=\frac{1}{\sqrt{2}}\left(|L, 2 \hbar\rangle+e^{i \pi}|R,-2 \hbar\rangle\right)=\frac{1}{\sqrt{2}}(|L, 2 \hbar\rangle-|R,-2 \hbar\rangle) .
$$

Using a half-wave plate, one can transform the states given by Eqs. (8) and (11) into the states

$$
\begin{aligned}
& |\Psi\rangle_{3}=\frac{1}{\sqrt{2}}(|R, 2 \hbar\rangle+|L,-2 \hbar\rangle), \\
& |\Psi\rangle_{4}=\frac{1}{\sqrt{2}}(|R, 2 \hbar\rangle-|L,-2 \hbar\rangle) .
\end{aligned}
$$

It is easily seen that the states $|\Psi\rangle_{2},|\Psi\rangle_{3}$ and $|\Psi\rangle_{4}$ are non-separable because the determinants of the relevant matrices are nonzero. Hence, the quantum states given by Eq. (8) and Eqs. (11)(13) represent the four maximum entangled Bell states.

\section{Conclusion}

We have demonstrated that the single-photon entanglement can be realized in case of a special kind of acousto-optic diffraction, a collinear acousto-gyration interaction in optical active and optically uniaxial (or isotropic) materials. Under these conditions, the orbital angular momentum in the diffracted beam must appear due to a general requirement of conservation of the total angular momentum. The entanglement between the two degrees of freedom, the SAM and the 
OAM, occurs when the standing longitudinal acoustic wave is excited in the medium. This makes it possible to realize the four maximum entangled Bell states. The criteria for verification of this type of entanglement are discussed.

\section{Acknowledgement}

The authors acknowledge the Ministry of Education and Science of Ukraine for financial support of the present study (the Project \#01118U003899).

\section{References}

1. Bokulich A and Jaeger G. Philosophy of quantum information and entanglement. Cambridge: Cambridge University Press (2010).

2. Einstein A, Podolsky B and Rosen N, 1935. Can quantum-mechanical description of physical reality be considered complete? Phys. Rev. 47: 777-780.

3. Bell J S, 1964. On the Einstein Podolsky Rosen paradox. Physics Physique Fizika. 1: 195200.

4. Freedman S J and Clauser J F, 1972. Experimental test of local hidden-variable theories. Phys. Rev. Lett. 28: 938-941.

5. Aspect A, Grangier P and Roger G, 1981. Experimental tests of realistic local theories via Bell's theorem. Phys. Rev. Lett. 47: 460-463.

6. Häffner H, Hänsel W, Roos C F, Benhelm J, Chek-al-kar D, Chwalla M, Körber T, Rapol U D, Riebe M, Schmidt P O, Becher C, Gühne O, Dür W and Blatt R, 2005. Scalable multiparticle entanglement of trapped ions. Nature. 438: 643-646.

7. Neumann P, Mizuochi N, Rempp F, Hemmer P, Watanabe H, Yamasaki S, Jacques V, Gaebel T, Jelezko F and Wrachtrup J, 2008. Multipartite entanglement among single spins in diamond. Science. 320: 1326-1329.

8. Zhao Z, Chen Y A, Zhang A N, Yang T, Briegel H J and Pan J W, 2004. Experimental demonstration of five-photon entanglement and open-destination teleportation. Nature. 430: 54-58.

9. Juan Yin, Ji-Gang Ren, He Lu, Yuan Cao, Hai-Lin Yong, Yu-Ping Wu, Chang Liu, ShengKai Liao, Fei Zhou, Yan Jiang, Xin-Dong Cai, Ping Xu, Ge-Sheng Pan, Hao Yin, Yu-Ao Chen, Cheng-Zhi Peng and Jian-Wei Pan, 2012. Quantum teleportation and entanglement distribution over 100-kilometre free-space channels. Nature. 488: 185-188.

10. Xi-Lin Wang, Xin-Dong Cai, Zu-En Su, Ming-Cheng Chen, Dian Wu, Li Li, Nai-Le Liu, Chao-Yang Lu and Jian-Wei Pan, 2015. Quantum teleportation of multiple degrees of freedom of a single photon. Nature. 518: 516-519.

11. Pittman T B, Jacobs B C and Franson J D, 2001. Probabilistic quantum logic operations using polarizing beam splitters. Phys. Rev. A. 64: 62311.

12. Kok P, Munro W J, Kae Nemoto, Ralph T C, Dowling J P and Milburn G J, 2007. Linear optical quantum computing with photonic qubits. Rev. Mod. Phys. 79: 135-174.

13. Ladd T D, Jelezko F, Laflamme R, Nakamura Y, Monroe C and O'Brien J L, 2010. Quantum computers. Nature. 464: 45-53.

14. Kwiat P G, Mattle K, Weinfurter H, Zeilinger A, Sergienko A V and Shih Y, 1995. New highintensity source of polarization-entangled photon pairs. Phys. Rev. Lett. 75: 4337-4341.

15. Boyer V, Marino A M, Pooser R C and Lett P D, 2008. Entangled images from four-wave mixing. Science. 321: 544-547.

Ukr. J. Phys. Opt. 2020, Volume 21, Issue 3 
16. Aspect A, Dalibard J and Roger G, 1982. Experimental test of Bell's inequalities using timevarying analyzers. Phys. Rev. Lett. 49: 1804-1807.

17. Aspect A, Grangier P and Roger G, 1982. Experimental realization of Einstein-PodolskyRosen-Bohm Gedankenexperiment: A new violation of Bell's inequalities. Phys. Rev. Lett. 49: 91-94.

18. Zwiebach B. 8.05 Quantum Physics II. Fall 2013. Massachusetts Institute of Technology: MIT OpenCourseWare, https://ocw.mit.edu. License: Creative Commons BY-NC-SA.

19. D’Ambrosio V, Carvacho G, Graffitti F, Vitelli C, Piccirillo B, Marrucci L and Sciarrino F, 2016. Entangled vector vortex beams. Phys. Rev. A. 94: 030304(R).

20. Lixiang Chen and Weilong She, 2010. Single-photon spin-orbit entanglement violating a Bell-like inequality. J. Opt. Soc. Amer. B. 27: A7-A10.

21. Aiello A, Töppel F, Marquardt C, Giacobino E and Leuchs G, 2015. Quantum-like nonseparable structures in optical beams. New J. Phys. 17: 043024.

22. Vlokh R, Vlokh O, Pyatak Yu and Skab I, 1990. Acoustogyration diffraction of light. Ferroelectrics. 110: 1117-1121.

23. Martynyuk-Lototska I Yu, Mys O G, Akimov S V, Krupych O M and Vlokh R O, 2010. Acoustogyration diffraction of optical waves: case of $\mathrm{SiO}_{2}$ and $\mathrm{TeO}_{2}$ crystals. Opto-Electron. Rev. 18: 137-149.

24. Skab I and Vlokh R, 2012. On the conservation of optical angular momentum acoustogyration diffraction of light Ukr. J. Phys. Opt. 13: 1-3.

Skab I., Kostyrko M. and Vlokh R. 2020. Single-photon entanglement at acousto-optic (acoustogyration) diffraction. Ukr.J.Phys.Opt. 21: 152 - 158. doi: 10.3116/16091833/21/3/152/2020

Анотація. Показано, що однофотонне заплутування можна реалізувати за умови колінеарної акустогіраиійної дифракиії в оптично активних $і$ оптично одновісних (або ізотропних) середовищах. Заплутування між двома ступенями вільності (спіновим моментом імпульсу і орбітальним моментом імпульсу) відбувається в тому разі, коли в зазначених середовищах збуджено стоячу поздовжню акустичну хвилю. Це дає змогу реалізувати чотири максимально заплутаних стани Белла. 